\title{
Molecular epidemiology of Orientia tsutsugamushi in chiggers and ticks from domestic rodents in Shandong, northern China
}

Meng Zhang ${ }^{1}$, Zhong-Tang Zhao ${ }^{1 *}$, Hui-Li Yang ${ }^{2}$, Ai-Hua Zhang ${ }^{2}$, Xing-Qu Xu ${ }^{3}$, Xiang-Peng Meng ${ }^{2}$, Hai-Yu Zhang ${ }^{3}$, Xian-Jun Wang ${ }^{4 *}$, Zhong Li ${ }^{4}$, Shu-Jun Ding ${ }^{4}$, Li Yang ${ }^{1}$ and Lu-Yan Zhang ${ }^{1}$

\begin{abstract}
Background: Scrub typhus is endemic to a $13,000,000-\mathrm{km}^{2}$ area of the Asia-Pacific region, and causes an annual incidence of 1 million people. The mortality rate of scrub typhus ranges from $6.1 \%$ to 25\% in Southeast Asia. Natural infection of Orientia tsutsugamushi has been identified in domestic rodents in Shandong Province. However, infestation of chiggers and ticks on the domestic rodents and prevalence and genotypes of 0 . tsutsugamushi in these Acarina remain unclear.
\end{abstract}

Methods: During September 2010 to March 2012, 3134 chiggers and 89 ticks were collected from domestic rodents captured in three counties of Shandong Province. We amplified and sequenced the 56-kDa type-specific antigen gene of 0 . tsutsugamushi from DNA samples of these Acarina and designated to genotype according to sequence analysis.

Results: Overall, the infestation rate of chiggers on domestic rodents was $17.0 \%$, and the chigger index was 5.38. The infestation rate of ticks on rodents was 3.1\%. Natural infection of O. tsutsugamushi was found in Leptotrombidium taishanicum, L. linhuaikongense, L. intermedium, L. scutellare, L. palpale, and Ixodes spp., the minimum positive rates of which were 5.9\%,3.2\%, 1.2\%, 0.8\%, 0.8\%, and 2.2\%, respectively. Kawasaki-like genotypes were predominant in chiggers and ticks on domestic rodents, which were detected from L. taishanicum, L. intermedium, L. scutellare, L. palpale, and Ixodes spp. Shimokoshi-like genotype was detected from L. palpale.

Conclusions: In the present study we investigated the infestation of chiggers and ticks on domestic rodents in Shandong Province, and identified the prevalence and genotypes of 0 . tsutsugamushi in the Acarina. Infestation of vector chiggers in domestic rodents, prevalence of 0 . tsutsugamushi in infested chiggers, and high nucleotide homologies among the $O$. tsutsugamushi sequences from the Acarina, their animal hosts and scrub typhus patients, implied that domestic rodents may play an important role in the transmission of scrub typhus in Shandong, China. Further studies are needed to verify the vector significance of chiggers and ticks that tested positive for 0 . tsutsugamushi, and to assess the risk of human exposure to chiggers and ticks on domestic rodents.

Keywords: Scrub typhus, Orientia tsutsugamushi, Chigger, Tick, Rodents

\footnotetext{
* Correspondence: ztzhao@sdu.edu.cn; xjwang62@163.com

${ }^{1}$ Department of Epidemiology and Health Statistics, School of Public Health,

Shandong University, Jinan, China

${ }^{4}$ Shandong Center for Disease Control and Prevention, Jinan, China

Full list of author information is available at the end of the article
} 


\section{Background}

Scrub typhus is an acute febrile disease caused by Orientia tsutsugamushi, which is transmitted by larval-stage trombiculid mites. It is endemic to a $13,000,000-\mathrm{km}^{2}$ area of the Asia-Pacific region, and causes an annual incidence of 1 million people [1,2]. The mortality rate of scrub typhus ranges from $6.1 \%$ to $25 \%$ in Southeast Asia $[3,4]$. Prevalence of scrub typhus and the burden of disease are usually underestimated. O. tsutsugamushi is a Gramnegative obligate intracellular bacterium categorized in the genus Orientia within the family Rickettsiaceae. In the recent decades, dramatic variation in phenotypes and genotypes of O. tsutsugamushi have been revealed in humans, animal hosts, and vector mites using immunological and molecular methods [5]. Highly polymorphic 56-kDa type-specific antigen (TSA), which consists of four variable domains (VD) I-IV presenting significant sequence heterogeneity among strains [6], is commonly used for type designation and evolutionary analysis of O. tsutsugamushi.

Scrub typhus has been endemic to southern China for decades, and it occurs mainly in summer and winter. The disease had not been reported in northern China until the first outbreak in Linyi City, Shandong Province in 1986 [7], and it occurs mainly in autumn and winter [8]; hitherto, sporadic cases and outbreaks have been reported nationwide [8]. According to the Shandong Diseases Reporting Information System, annual reported cases of scrub typhus have continuously increased in the past few years. In Shandong Province, the annual incidence of scrub typhus has increased from 0.23 in 2006 to 0.64 in 2012 per 100,000 people.

It was indicated that improper behavior at outdoor activities were responsible for scrub typhus $[9,10]$. Therefore, many researches focused on the prevalence of O. tsutsugamushi in chiggers parasitizing on wild rodents. The rate of positivity for O. tsutsugamushi ranged from $0.9 \%$ to $5.7 \%$ in chiggers collected from wild rodents [11-13]. A case-control study conducted in Shandong Province revealed that the living environment of poor sanitary conditions was significantly associated with the risk of scrub typhus [14]. In our previous study, we identified the prevalence of O. tsutsugamushi in domestic rodents, and proposed the hypothesis that domestic rodents may play an important role in the transmission of scrub typhus [15]. However, infestation of chiggers and ticks on domestic rodents and prevalence and genotype of $O$. tsutsugamushi in these Acarina remain unclear. To verify the hypothesis and provide a better understanding of the ecology and epidemiology of O. tsutsugamushi, chiggers and ticks collected from domestic rodents require screening for O. tsutsugamushi. In this study, the $56-\mathrm{kDa}$ TSA gene of O. tsutsugamushi was amplified and sequenced from chiggers and ticks parasitizing on domestic rodents, to investigate the host range of O. tsutsugamushi, identify prevalent genotypes of $O$. tsutsugamushi in vectors, and evaluate the transmission risk of scrub typhus from a chigger or tick bite.

\section{Methods}

\section{Study sites and ectoparasite collection}

Shandong Province, located at the eastern coast of China, was a typical epidemic area of scrub typhus. It has a temperate and monsoonal climate. Xintai, Daiyue, and Yinan, characterized by mountains and hills, in middle Shandong were selected for investigation. Rodents were trapped from residences of farmers in rural areas every season from September 2010 to March 2012. Species of the captured mammals were identified and labeled. For each mammal, chiggers and ticks were removed from the ears, washed with TE buffer, and identified to species based on general features of external morphology.

\section{DNA preparation}

We pooled 10-50 chiggers or 5-30 ticks of the same species collected from one animal host for homogenization

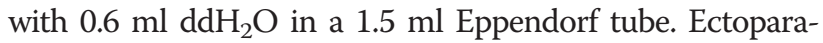
sites of the same species from several animals of the same species at the same site and date were put together to make sure no less than 10 chiggers or 5 ticks were in a pool. The homogenates were added with 1/10 volume of lysis buffer (100 mM Tris- $\mathrm{HCl}, \mathrm{pH}$ 8.0, $10 \mathrm{mM}$ EDTA, $10 \%$ SDS) and $20 \mu \mathrm{L}$ Proteinase $\mathrm{K}$, and inoculated at $55^{\circ} \mathrm{C}$ for $1 \mathrm{hr}$. DNA was then extracted with equal volumes of phenol chloroform isoamylol (25:24:1) twice and precipitated by ethanol. After washing with $75 \%$ ethanol and drying, DNA was dissolved in $30 \mu \mathrm{L}$ TE buffer (10 mM Tris-HCl, $1 \mathrm{mM}$ EDTA, $\mathrm{pH}$ 8.0) and stored at $-20^{\circ} \mathrm{C}$ for later usage.

\section{Nested polymerase chain reaction}

Coding sequences covering VD I-III of $56-\mathrm{kDa}$ TSA were amplified using nested PCR. Two sets of primers used were as follows: outer primers, comprising 34 (5'TCAAGCTTATTGCTAGTGCAATGTCTGC-3') and 55 (5'-AGGGATCCCTGCTGCTGTGCTTGCTGCG-3') [16], and inner primers, comprising E (5'-GTTGGAGGAAT GATTACTGG-3') and B (5'-ACAGATGCACTATTAGG CAA-3') [17].

The initial round of PCR was started with a 5-min denaturation at $94^{\circ} \mathrm{C}$, followed by 30 cycles of $95^{\circ} \mathrm{C}$ for $30 \mathrm{sec}, 57^{\circ} \mathrm{C}$ for $2 \mathrm{~min}, 70^{\circ} \mathrm{C}$ for $2 \mathrm{~min}$, and then a final extension at $72^{\circ} \mathrm{C}$ for $10 \mathrm{~min}$. The product was used as the template for the second round of PCR, which was started with a 3-min denaturation at $95^{\circ} \mathrm{C}$, followed by 30 cycles of $95^{\circ} \mathrm{C}$ for $30 \mathrm{sec}, 55^{\circ} \mathrm{C}$ for $30 \mathrm{sec}, 72^{\circ} \mathrm{C}$ for $1 \mathrm{~min}$, and then a final extension at $72^{\circ} \mathrm{C}$ for $10 \mathrm{~min}$. The nested PCR products were visualized with an ultraviolet transilluminator after agarose gel electrophoresis 
and staining with ethidium bromide. Amplicons with a length of $\approx 733 \mathrm{bp}$ were purified with Gel Extraction Kit (Omega, Norcross, GA, USA). DNA sequencing was carried out in an ABI 3730xl DNA Analyzer (Biosune, Shanghai, China).

\section{Phylogenetic analysis and sequence homologies}

Complete or partial sequences encoding 56-kDa TSA of $O$. tsutsugamushi reference strains were retrieved from the GenBank (Table 1). Multiple sequence alignment was performed with Mega software using ClustalW algorithm. Phylogenetic relationships between O. tsutsugamushi strains from this study and the reference strains were inferred with Mega software by neighbor-joining method. Reliability of the phylogenetic analysis was evaluated using the bootstrap test on 1,000 replicates. Sequence homologies were calculated using MegAlign of Lasergene software (DNASTAR Inc., Madison, WI, USA).

\section{Nucleotide sequence accession numbers}

DNA sequences of $O$. tsutsugamushi obtained in this study were deposited into GenBank under the accession no. KC456648-KC456650, KC456657- KC456660, and KF421129 (Table 2).

Table 1 Reference sequences of Orientia tsutsugamushi used in the phylogenetic analysis

\begin{tabular}{|c|c|c|c|c|c|}
\hline Isolate & Source & Accession no. & Country & Year & Length (bp) \\
\hline Kato & Human & MM63382 & Japan & 1955 & 1590 \\
\hline Taiwan CDC Gilliam & - & DQ485289 & Taiwan & - & 1860 \\
\hline Karp & Human & M33004 & New Guinea & 1943 & 1599 \\
\hline Kuroki & Human & M63380 & Japan & 1981 & 1599 \\
\hline Kawasaki & Human & M63383 & Japan & 1981 & 1569 \\
\hline TA678 & Rattus rattus & U19904 & Thailand & 1963 & 1548 \\
\hline TA763 & R. rattus & U80636 & Thailand & 1963 & 1581 \\
\hline TA686 & Tupaia glis & U80635 & Thailand & 1963 & 1599 \\
\hline TA716 & Menetes berdmorei & U19905 & Thailand & 1963 & 1575 \\
\hline Shimokoshi & Human & M63381 & Japan & 1980 & 1565 \\
\hline Boryong & Human & L04956 & South Korea & 1998 & 1602 \\
\hline HSB1 & Rodent & AF302983 & Japan & 1996-1997 & 1454 \\
\hline Yonchon & Human & U19903 & South Korea & 1989 & 1551 \\
\hline LX-1 & Leptotrombidium spp. & AF173042 & Japan & 1986 & 1445 \\
\hline LC-1 & L. chiangraiensis & FJ374771 & Thailand & 2008 & 1590 \\
\hline LA-1 & L. arenicola & AF173049 & Malaysia & 1993 & 1432 \\
\hline LF-1 & L. fletcheri & AF173050 & Malaysia & 1993 & 1428 \\
\hline Fuji & L. fuji & AF201834 & Japan & 1998 & 1431 \\
\hline Sxh951 & Human & AF050669 & China & 1998 & 1544 \\
\hline Neimeng-65 & Cricetulus barabansis & DQ514319 & China & 2004-2005 & 1535 \\
\hline Shandong-XDM2 & L. scutellare & DQ514320 & China & 1996 & 1591 \\
\hline $\mathrm{CHL}$ & Human & JX202567 & China & 2011 & 658 \\
\hline ZQL & Human & JX202576 & China & 2011 & 657 \\
\hline ZZF & Human & KC456647 & China & 2011 & 655 \\
\hline STAD10-24 & R. norvegicus & JX202582 & China & 2010 & 656 \\
\hline STAD10-792 & Mus musculus & JX202583 & China & 2010 & 689 \\
\hline TAXT11-1023 & M. musculus & JX202584 & China & 2011 & 660 \\
\hline TAXT11-1103 & M. musculus & JX202585 & China & 2011 & 706 \\
\hline TADY11-1168 & M. musculus & JX202586 & China & 2011 & 676 \\
\hline TADY12-0305 & R. norvegicus & JX202587 & China & 2012 & 661 \\
\hline TADY12-0307 & M. musculus & JX202588 & China & 2012 & 660 \\
\hline YN11-24 & C. barabansis & KC456651 & China & 2011 & 667 \\
\hline
\end{tabular}


Table 2 Sequences encoding partial 56-kDa type-specific antigen of Orientia tsutsugamushi determined in this study

\begin{tabular}{|c|c|c|c|c|c|}
\hline Sample code & Source & Accession no. & Location & Sampling date & Length (bp) \\
\hline YNM1 & L. palpale & KC456648 & Yinan & Mar-2011 & 662 \\
\hline YNM2 & L. intermedium & KC456649 & Yinan & Jan-2011 & 666 \\
\hline YNM5 & L. taishanicum & KC456650 & Yinan & Jan-2011 & 669 \\
\hline TAM1 & L. palpale & KC456657 & Tai'an & Oct-2010 & 657 \\
\hline TAM2 & L. palpale & KC456658 & Tai'an & Oct-2010 & 669 \\
\hline TAM3 & L. palpale & KC456659 & Tai'an & Oct-2011 & 662 \\
\hline TAM5 & L. scutellare & KC456660 & Tai'an & Oct-2011 & 656 \\
\hline TAT1 & Ixodes spp. & KF421129 & Tai'an & Oct-2011 & 657 \\
\hline
\end{tabular}

\section{Ethical statement}

The study was approved by the Ethics Committee on Preventive Medicine of Shandong University.

\section{Results}

Infestation of Acarina on domestic rodents

During the study period, 583 domestic rodents were captured, including 359 Rattus norvegicus, 222 Mus musculus, and 2 Rattus rattus. We collected 3134 chigger mites from the ears of the captured mammals, including L. taishanicum (34, 1.1\%), L. linhuaikongense (62, $2.0 \%)$, L. intermedium (817, 26.1\%), L. scutellare (608, 19.4\%), L. palpale (1275, 40.7\%), L. laxoscutum (46, 1.5\%), Walchia pacifica (112, 3.6\%), Odontacarus majesticus (177, 5.6\%), and Gahrliepia octosetosa (3, 0.1\%). Rates of chigger infestation on domestic rodents in spring, summer, autumn and winter were $4.7 \%, 15.4 \%, 27.0 \%$, and $10.4 \%$, respectively (Table 3 ). Seasonal fluctuation of chigger mites during the four seasons was shown in Figure 1. L. palpale, L. intermedium, and L. scutellare were predominant chigger species in autumn and winter, while $O$. majesticus and $W$. pacifica were predominant in summer. Overall, the infestation rate of chigger mites on the captured domestic rodents was $17.0 \%$, and the chigger index was 5.38 . Among the $359 R$. norvegicus, 72 (20.1\%) were found to be parasitized by chiggers, with a chigger index of 8.73 . The infestation rate of chiggers in $M$. musculus was $12.2 \%$, and the chigger index was 2.74 . Chigger densities of different species collected from different hosts were demonstrated in Table 4. L. intermedium, L. scutellare, and $L$. palpale were the first three predominant species of chiggers from both $R$. norvegicus and $M$. musculus. No chigger was examined from $R$. rattus. In addition, 89 ticks of Ixodes spp. were collected from 18 rodents. The infestation rate of ticks on domestic rodents was $3.1 \%$.

\section{Prevalence of 0 . tsutsugamushi in chiggers and ticks}

One hundred and sixty four pools of mites were screened for O. tsutsugamushi using nested PCR. Among the 29 positive mite homogenates determined by nested PCR, 24 pools were collected from $R$. norvegicus, and 5 from M. musculus. Natural infection of O. tsutsugamushi was found in L. taishanicum, L. linhuaikongense, L. intermedium, L. scutellare, and L. palpale, the minimum positive rates (MPR = positive pools/total chigger counts) of which were $5.9 \%, 3.2 \%, 1.2 \%, 0.8 \%$, and $0.8 \%$, respectively. The MPRs of chigger mites in spring, summer, autumn, and winter were $10 \%, 0,0.9 \%$, and $1.8 \%$, respectively (Table 5). The collected ticks were divided into 12 pools, of which 2 pools were positive for O. tsutsugamushi.

\section{Phylogenetic analysis and sequence homologies}

Seven sequences of $O$. tsutsugamushi from chiggers and 1 from ticks determined in this study, 11 sequences previously determined in patients and domestic rodents in the same study area, and 21 reference sequences of O. tsutsugamushi were enrolled in the phylogenetic analysis. The O. tsutsugamushi harbored by chigger mites were related to either Kawasaki or Shimokoshi strain originating from Japan (Figure 2). TAM1 had 99.4\% nucleotide identity to Shimokoshi strain, and denominated as SKS genotype. TAM5, YNM1, YNM2, YNM5, and

Table 3 Rates of chigger infestation on domestic rodents, northern China

\begin{tabular}{lccccc}
\hline Mammal species & \multicolumn{5}{c}{ Season, no. infested/no. captured (\%) } \\
\cline { 2 - 6 } & Spring & Summer & Autumn & Winter & Total \\
\hline Rattus norvegicus & $4 / 99(4.0)$ & $12 / 52(23.1)$ & $49 / 151(32.5)$ & $7 / 57(12.3)$ & $72 / 359(20.1)$ \\
Mus musculus & $2 / 29(6.9)$ & $0 / 26(0)$ & $19 / 101(18.8)$ & $6 / 66(9.1)$ & $27 / 222(12.2)$ \\
R. rattus & 0 & 0 & 0 & $0 / 2(0)$ & $0 / 2(0)$ \\
Total & $6 / 128(4.7)$ & $12 / 78(15.4)$ & $68 / 252(27.0)$ & $13 / 125(10.4)$ & $99 / 583(17.0)$ \\
\hline
\end{tabular}




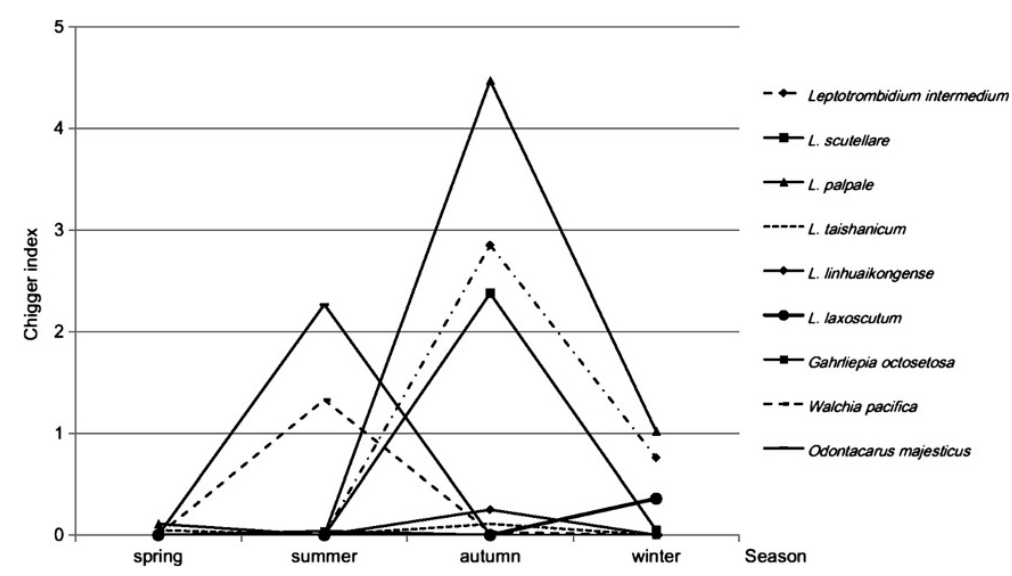

Figure 1 Seasonal fluctuation of chigger mites on domestic rodents in Shandong, northern China.

TAT1 were identical to CHL (KWS1 genotype), which had $96.5 \%$ nucleotide identity to Kawasaki strain. TAM2 and TAM3 were identical to STAD10-24 (KWS2 genotype), which had an identity of $96.4 \%$ to Kawasaki strain. The nucleotide sequence identities ranged from $70.7 \%$ to $100 \%$ among the 8 sequences. The O. tsutsugamushi sequences determined from chiggers and ticks had identities ranging from $70.5 \%$ to $100 \%$ with those previously identified in humans, and $69.6 \%$ to $100 \%$ with those in domestic rodents in the study area (Table 6). TAM2, which was identified from the chiggers parasitizing on R. norvegicus STAD10-24, was identical to O. tsutsugamushi sequence carried by the same rodent. TAM5, which was identified in the mite pools collected from M. musculus TAXT11-1023, showed 99.9\% identity with O. tsutsugamushi sequence carried by the same rodent. TAM3 detected in chiggers and TAT1 in ticks, which were collected from the same rodent, had an identity of $99.9 \%$ with each other. The nucleotide homologies of partial 56-kDa TSA gene between $O$. tsutsugamushi determined in this study and reference strains were shown in Table 6.

\section{Discussion}

Vectors for O. tsutsugamushi require four prerequisites: 1) epidemiological correlation with the occurrence of scrub typhus; 2) natural infection of O. tsutsugamushi; 3) could feed on the hosts and transmit the agent; and 4) transovarial transmission of the agent. Six species of chigger mites has been confirmed as the vectors for O. tsutsugamushi in China, which were Leptotrombidium deliense, L. insularae, L. kaohuensis, L. rubellum, L. jishoum, and L. scutellare [18]. O. tsutsugamushi DNA was detected in L. taishanicum, L. linhuaikongense, L. intermedium, L. scutellare, L. palpale and Ixodes spp. in this study. To our knowledge, it is the first study to identify O. tsutsugamushi in L. taishanicum and Ixodes spp.. It was reported that

Table 4 Species of chiggers collected from domestic rodents, northern China

\begin{tabular}{|c|c|c|c|c|}
\hline \multirow[t]{2}{*}{ Chigger species } & \multicolumn{4}{|c|}{ Mammal species, no. chiggers (chigger index) } \\
\hline & $\begin{array}{l}\text { Rattus norvegicus } \\
\qquad(\mathrm{n}=359)\end{array}$ & $\begin{array}{l}\text { Mus musculus } \\
\qquad(\mathrm{n}=222)\end{array}$ & $\begin{array}{l}\text { R. rattus } \\
(n=2)\end{array}$ & $\begin{array}{c}\text { Total } \\
(n=586)\end{array}$ \\
\hline Leptotrombidium intermedium & $601(1.67)$ & $216(0.97)$ & 0 & $817(1.39)$ \\
\hline L. scutellare & $426(1.19)$ & $182(0.82)$ & 0 & $608(1.04)$ \\
\hline L. palpale & $1078(3.00)$ & $197(0.89)$ & 0 & $1275(2.18)$ \\
\hline L. taishanicum & $30(0.08)$ & $4(0.02)$ & 0 & $34(0.06)$ \\
\hline L. linhuaikongense & $62(0.17)$ & 0 & 0 & $62(0.11)$ \\
\hline L. laxoscutum & $42(0.12)$ & $4(0.02)$ & 0 & $46(0.08)$ \\
\hline Gahrliepia octosetosa & $3(0.01)$ & 0 & 0 & $3(0.01)$ \\
\hline Walchia pacifica & $106(0.30)$ & $6(0.03)$ & 0 & $112(0.19)$ \\
\hline Odontacarus majesticus & $177(0.49)$ & 0 & 0 & $177(0.30)$ \\
\hline Total & $2525(7.03)$ & 609 (2.74) & 0 & $3134(5.35)$ \\
\hline
\end{tabular}


Table 5 Minimum positive rate of Orientia tsutsugamushi in chigger mites collected from domestic rodents, northern China

\begin{tabular}{lccccc}
\hline Chigger species & \multicolumn{3}{c}{ Season, no. positive pools/total counts (\%) } \\
\cline { 2 - 5 } & Spring & Summer & Autumn & Winter & Total \\
\hline Leptotrombidium intermedium & 0 & 0 & $9 / 721(1.2)$ & $1 / 96(1.0)$ & $10 / 817(1.2)$ \\
L. scutellare & 0 & 0 & $4 / 602(0.7)$ & $1 / 6(16.7)$ & $5 / 608(0.8)$ \\
L. palpale & $1 / 14(7.1)$ & 0 & $6 / 1132(0.5)$ & $3 / 129(2.3)$ & $10 / 1275(0.8)$ \\
L. taishanicum & $1 / 6(16.7)$ & 0 & $1 / 28(3.6)$ & 0 & $2 / 34(5.9)$ \\
L. linhuaikongense & 0 & 0 & $2 / 62(3.2)$ & 0 & $2 / 62(3.2)$ \\
L. laxoscutum & 0 & 0 & 0 & 0 & 0 \\
Gahrliepia octosetosa & 0 & $0 / 3(0)$ & 0 & $0 / 3(0)$ \\
Walchia pacifica & 0 & $0 / 104(0)$ & $0 / 8(0)$ & 0 & $0 / 112(0)$ \\
Odontacarus majesticus & 0 & $0 / 177(0)$ & 0 & $0 / 177(0)$ \\
Total & $2 / 20(10.0)$ & $0 / 284(0)$ & $22 / 2553(0.9)$ & $5 / 277(1.8)$ & $29 / 3134(0.9)$ \\
\hline
\end{tabular}

O. tsutsugamushi had been successfully isolated from Ixodes granulatus collected from domestic rodents; however, artificial infection experiments showed that the agent could live for no more than 4 days in the ticks [18]. It was possible that some species of $O$. tsutsugamushi-positive chiggers and ticks acquired the agent from infected hosts, but were not able to support their survival or proliferation.

We demonstrated that three genotypes of O. tsutsugamush $i$ were circulating in the chiggers and ticks collected from domestic rodents in Shandong Province, which were KWS1, KWS2, and SKS. KWS1 genotype was detected from L. taishanicum, L. intermedium, L. scutellare, L. palpale, and Ixodes spp.. KWS2 genotype was identified in L. scutellare and L. palpale. And SKS genotype was found in L. palpale. O. tsutsugamushi DNA was detected from two pools of L. linhuaikongense; however, we failed to identify the genotypes because of unsuccessful sequencing.

The majority of scrub typhus cases occurred in October and November in Shandong Province [8]. L. palpale, L. intermedium, and L. scutellare were predominant mite species parasitizing on domestic rodents during autumn in Shandong. L. scutellare has been confirmed to be a vector for scrub typhus. KWS1 genotype of O. tsutsugamushi was detected from L. intermedium; however, the low infestation rate to humans makes it of less significance as a vector for scrub typhus. Genotypes of O. tsutsuga$m u s h i$ are usually restricted to a specific chigger species [19]. L. palpale could be infested with KWS1, KWS2, and SKS genotypes of O. tsutsugamushi, and may be a possible vector of public health importance. Odontacarus majesticus and Walchia pacifica were predominant during the summer. It was reported that Odontacarus majesticus and Walchia pacifica could be infested with O. tsutsugamushi in China [20,21]. Scrub typhus cases were sporadically reported in summer in Shandong Province.
Although no O. tsutsugamushi DNA was detected from the two species of chiggers in this study, their potential to be vectors for in-house transmission of scrub typhus should not be excluded. The vector competence of the above mentioned $O$. tsutsugamushi-positive chiggers and ticks requires further evaluation.

Shimokoshi-type O. tsutsugamushi presented weak virulence to mice [22]; however, the resulting clinical manifestations in humans need more documentation. Scrub typhus cases of Shimokoshi type were first reported in Niigata Prefecture, Japan in 1984 [22], and recently reported in northeastern and western Japan [23]. Hitherto, scrub typhus cases caused by Shimokoshi-like genotypes of O. tsutsugamushi have not been reported in China. Detection of Shimokoshi-like genotype of O. tsutsugamushi in L. palpale is an alert to potential scrub typhus outbreaks of this genotype in humans. It was reported that Shimokoshi type had low cross reactivity with the prototype strains of Kato, Karp, and Gilliam [22]. Currently used diagnostic reagents of scrub typhus in China, with the three prototype strains as antigens, is not adequate to detect Shimokoshi-type $O$. tsutsugamushi. In order to identify the clinical profile of scrub typhus cases caused by Shimokoshi-like genotypes of O. tsutsugamushi, Shimokoshi strain should be added in the antigen pool of diagnostic tests.

Rodents were found to be active in the living room, kitchen, toilet, and yard in the study area. Natural infection of O. tsutsugamushi was identified in domestic rodents captured in Shandong, China [15]. O. tsutsugamushi DNA was detected in Acarina parasitizing on domestic rodents, and had high identities with those detected in the domestic rodents and in scrub typhus patients. It was indicated that chiggers had the opportunity to get infected from the domestic rodents, be taken to wherever the rodents go, and transmit the agent to their offspring. The residents could be infected with scrub 


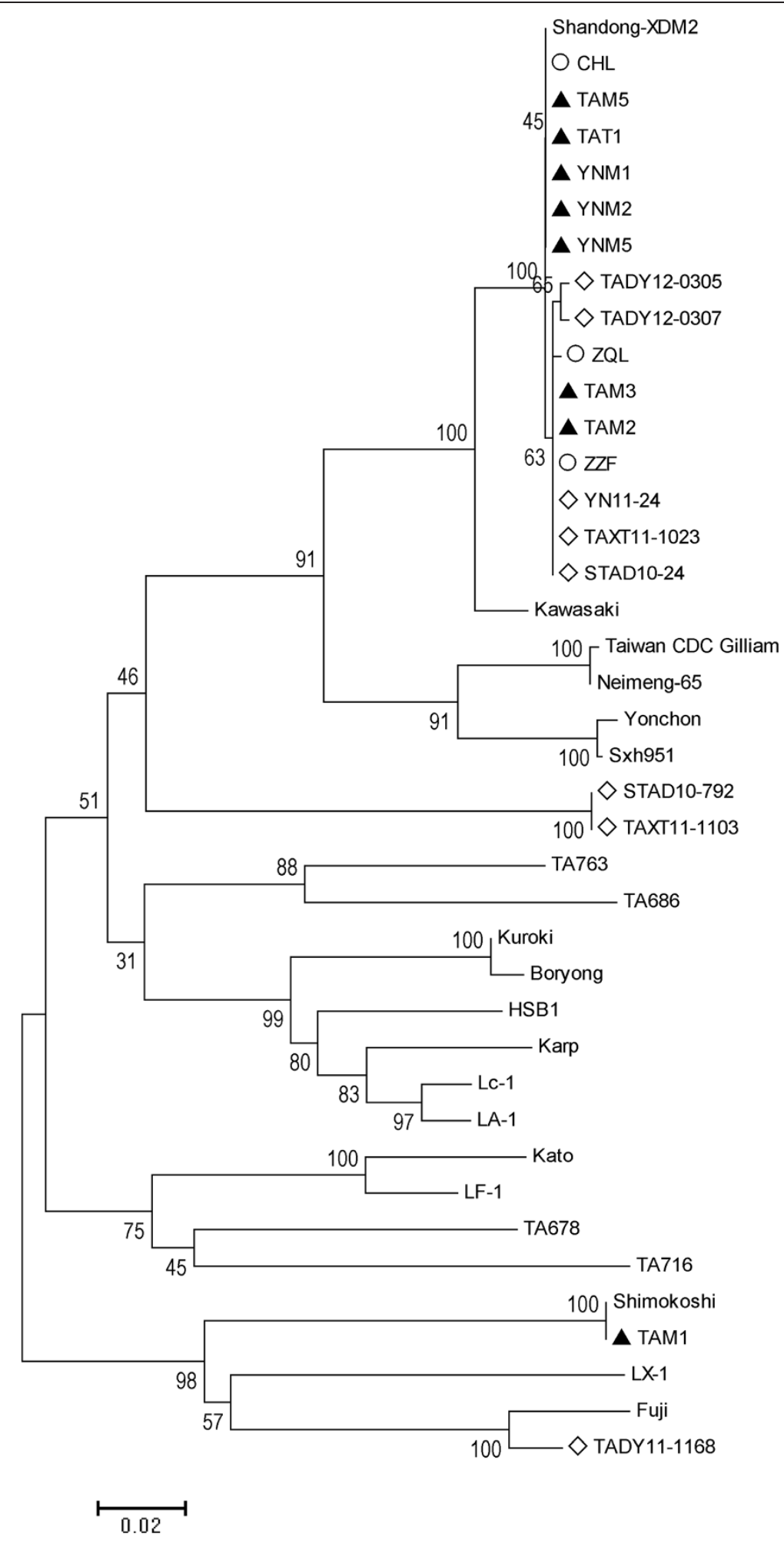

Figure 2 Phylogenetic tree based on partial 56-kDa type-specific antigen gene of Orientia tsutsugamushi. The phylogenetic analysis was performed using the neighbor-joining method with Kimura 2-parameter distance model. Solid triangle indicates sequences determined in this study. Hollow circle and diamond indicate sequences previously determined in scrub typhus patients and domestic rodents in the study area. Scale bar indicates genetic distance. 
Table 6 Nucleotide homologies of partial 56-kDa TSA gene between Orientia tsutsugamushi strains

\begin{tabular}{|c|c|c|c|c|c|c|c|c|}
\hline \multirow[t]{2}{*}{ Reference strain } & \multicolumn{8}{|c|}{ Homologies (\%) } \\
\hline & TAM1 & TAM2 & TAM3 & TAM5 & YNM1 & YNM2 & YNM5 & TAT1 \\
\hline Kawasaki & 69.8 & 96.4 & 96.4 & 96.5 & 96.5 & 96.5 & 96.5 & 96.5 \\
\hline Shimokoshi & 99.4 & 70.4 & 70.4 & 70.4 & 70.4 & 70.4 & 70.4 & 70.4 \\
\hline Shandong-XDM2 & 71.2 & 99.9 & 99.9 & 100 & 100 & 100 & 100 & 100 \\
\hline $\mathrm{CHL}$ & 70.7 & 99.9 & 99.9 & 100 & 100 & 100 & 100 & 100 \\
\hline ZQL & 70.5 & 99.9 & 99.9 & 99.7 & 99.7 & 99.7 & 99.7 & 99.7 \\
\hline ZZF & 70.7 & 100 & 100 & 99.9 & 99.9 & 99.9 & 99.9 & 99.9 \\
\hline TADY12-0305 & 70.3 & 99.6 & 99.6 & 99.4 & 99.4 & 99.4 & 99.4 & 99.4 \\
\hline TADY12-0307 & 70.4 & 99.6 & 99.6 & 99.4 & 99.4 & 99.4 & 99.4 & 99.4 \\
\hline YN11-24 & 70.5 & 99.9 & 99.9 & 99.7 & 99.7 & 99.7 & 99.7 & 99.7 \\
\hline TAXT11-1023 & 70.7 & 100 & 100 & 99.9 & 99.9 & 99.9 & 99.9 & 99.9 \\
\hline STAD10-24 & 70.7 & 100 & 100 & 99.9 & 99.9 & 99.9 & 99.9 & 99.9 \\
\hline STAD10-792 & 69.6 & 76.4 & 76.4 & 76.4 & 76.4 & 76.4 & 76.4 & 76.4 \\
\hline TAXT11-1103 & 69.6 & 76.4 & 76.4 & 76.4 & 76.4 & 76.4 & 76.4 & 76.4 \\
\hline TADY11-1168 & 75.7 & 70.0 & 70.0 & 70.0 & 70.0 & 70.0 & 70.0 & 70.0 \\
\hline
\end{tabular}

typhus through occasional bites of the infected chiggers. Conclusions of the present study supported our previous hypothesis that domestic rodents play an important role in the transmission of scrub typhus.

However, the results should be interpreted under some limitations. First, chiggers usually parasitize on the whole body surface of animal hosts, especially on ears, breasts, genitals, and anus; however, only those that were parasitizing on the ears were collected in this study. The actual infestation of chiggers on domestic rodents and chigger index were likely to be larger than those indicated in the study. Second, considering the copies of O. tsutsugamushi from individual chigger were too low for PCR detection, we screened for O. tsutsugamushi from chigger pools rather than individual mite. The actual rate of positivity for O. tsutsugamushi in chiggers was no less than that indicated by MPR. Besides, there is a possibility that more than one genotype strain of O. tsutsugamushi existing in a pool, but only the predominant type could be detected in the study. Third, we failed to identify the collected ticks into species, which is required in future research.

\section{Conclusions}

In the present study, we identified the prevalence of O. tsutsugamushi in chiggers and ticks collected from domestic rodents in Shandong, China. High nucleotide homologies were found among the O. tsutsugamushi sequences from the collected Acarina, their animal hosts and scrub typhus patients. The results indicated a possibility that chiggers and ticks acquire O. tsutsugamushi from the domestic rodents, and transmit it to humans, which supported our hypothesis that domestic rodents may play an important role in the transmission of scrub typhus in Shandong, China. Deracination inside the residences would be a necessary and effective measure to reduce the transmission risk of scrub typhus. Further studies are needed to verify the vector significance of chiggers and ticks that tested positive for O. tsutsugamushi, and to assess the risk of human exposure to chiggers and ticks on domestic rodents.

\section{Abbreviations}

TSA: Type-specific antigen; VD: Variable domain; O. tsutsugamushi: Orientia tsutsugamushi; R. rattus: Rattus rattus; M. musculus: Mus musculus;

R. norvegicus: Rattus norvegicus; L. taishanicum: Leptotrombidium taishanicum; L. intermedium: Leptotrombidium intermedium; L. scutellare: Leptotrombidium scutellare; L. palpale: Leptotrombidium palpale; L. linhuaikongense: Leptotrombidium linhuaikongense; L. laxoscutum: Leptotrombidium laxoscutum;

W. pacifica: Walchia pacifica; O. majesticus: Odontacarus majesticus; and

G. octosetosa: Gahrliepia octosetosa.

Competing interests

The authors declare that they have no competing interests.

Authors' contributions

$M Z, Z T Z$, and XJW were responsible for the conception and design of the study. MZ, ZL, HLY, AHZ, XPM, XQX, HYZ, and SJD carried out rodent capture, ectoparasite collection, and morphological identifications of chiggers and ticks. MZ, LY, and LYZ carried out detection of O. tsutsugamushi and bioinformatics process. All the authors participated in manuscript drafting, and approved the final manuscript.

\section{Acknowledgements}

The work was supported by grants from the National Natural Science Foundation of China (No. 81273133, 30972515). The funders had no role in study design, data collection and analysis, decision to publish, or preparation of the manuscript. 


\section{Author details}

${ }^{1}$ Department of Epidemiology and Health Statistics, School of Public Health, Shandong University, Jinan, China. ${ }^{2}$ Tai'an Center for Disease Control and Prevention, Tai'an, China. ${ }^{3}$ Yinan Center for Disease Control and Prevention, Linyi, China. ${ }^{4}$ Shandong Center for Disease Control and Prevention, Jinan, China.

Received: 12 August 2013 Accepted: 20 October 2013

Published: 29 October 2013

\section{References}

1. Oaks SCJ, Ridway RL, Shirai A, Twartz JC: Scrub typhus. Inst Med Res Malays Bull 1983, 21:1-98.

2. Watt G, Parola P: Scrub typhus and tropical rickettsioses. Curr Opin Infect Dis 2003, 16(5):429-436.

3. Lee CS, Hwang JH, Lee HB, Kwon KS: Risk factors leading to fatal outcome in scrub typhus patients. Am J Trop Med Hyg 2009, 81(3):484-488.

4. Wang CC, Liu SF, Liu JW, Chung YH, Su MC, Lin MC: Acute respiratory distress syndrome in scrub typhus. Am J Trop Med Hyg 2007, 76(6):1148-1152.

5. Kelly DJ, Fuerst PA, Ching WM, Richards AL: Scrub typhus: the geographic distribution of phenotypic and genotypic variants of Orientia tsutsugamushi. Clin Infect Dis 2009, 48(Suppl 3):S203-S230.

6. Ohashi N, Nashimoto H, Ikeda H, Tamura A: Diversity of immunodominant 56-kDa type-specific antigen (TSA) of Rickettsia tsutsugamushi. Sequence and comparative analyses of the genes encoding TSA homologues from four antigenic variants. J Biol Chem 1992, 267(18):12728-12735.

7. $\quad$ Yang YF, Wang $J$, Yao YC: Investigation of the first scrub typhus epidemic in Shandong Province. Chin J Epidemiol 1987, 8(5):280.

8. Zhang M, Zhao ZT, Wang XJ, Li Z, Ding L, Ding SJ: Scrub typhus: surveillance, clinical profile and diagnostic issues in Shandong, China. Am J Trop Med Hyg 2012, 87(6):1099-1104.

9. Kweon SS, Choi JS, Lim HS, Kim JR, Kim KY, Ryu SY, Lee SD, Im HK, Kwon JW: A community-based case-control study of behavioral factors associated with scrub typhus during the autumn epidemic season in South Korea. Am J Trop Med Hyg 2009, 80(3):442-446.

10. Lyu Y, Tian L, Zhang L, Dou X, Wang X, Li W, Zhang X, Sun Y, Guan Z, Li X, et al: A case-control study of risk factors associated with scrub typhus infection in Beijing, China. PLoS One 2013, 8(5):e63668.

11. Lee HI, Shim SK, Song BG, Choi EN, Hwang KJ, Park MY, Park C, Shin EH: Detection of Orientia tsutsugamushi, the causative agent of scrub typhus, in a novel mite species, Eushoengastia koreaensis, in Korea. Vector Borne Zoonotic Dis 2011, 11(3):209-214.

12. Shim SK, Choi EN, Yu KO, Park HJ, Kim CM, Lee KH, Park JK, Park PH, Yoon MH Park SH, et al: Characterisation of Orientia tsutsugamushi genotypes from wild rodents and chigger mites in Korea. Clin Microbiol Infect 2009, 15(Suppl 2):311-312.

13. Takahashi M, Misumi H, Urakami H, Nakajima S, Furui S, Yamamoto S, Furuya Y, Misumi M, Matsumoto I: Mite vectors (Acari: Trombiculidae) of scrub typhus in a new endemic area in northern Kyoto, Japan. J Med Entomol 2004, 41(1):107-114.

14. Ding L, Wang XJ, Li Z, Ding SJ, Zhang M, Zhao ZT: Epidemic characteristics and related factors of autumn-winter type scrub typhus in Shandong area, 2010. Chin J Publ health 2013, 29(4):543-545.

15. Zhang M, Zhao ZT, Wang XJ, Li Z, Ding L, Ding SJ, Yang HL: Genetic variants of orientia tsutsugamushi in Domestic Rodents, Northern China. Emerg Infect Dis 2013, 19(7):1135-1137.

16. Furuya $Y$, Yoshida Y, Katayama T, Yamamoto S, Kawamura A Jr: Serotype-specific amplification of Rickettsia tsutsugamushi DNA by nested polymerase chain reaction. J Clin Microbiol 1993, 31(6):1637-1640.

17. Enatsu T, Urakami H, Tamura A: Phylogenetic analysis of Orientia tsutsugamushi strains based on the sequence homologies of 56-kDa type-specific antigen genes. FEMS Microbiol Lett 1999, 180(2):163-169.

18. Yu ES, Chen XR, Wu GH, Guo HB: Study on scrub typhus in China. 2000:13-15.

19. Kawamura A, Tanaka H, Tamura A: Tsutsugamushi disease. Tokyo (Japan): University of Tokyo Press; 1995.

20. Li JC, Zheng XY, Xi ZY, Ni H, Zhang HH, Chen CF: Basic studies on trombiculid mites and vector chiggers mites in the transmission of tsutsugamushi disease for 45 years. Acad J SUMS 2002, 23(1):1-9.
21. Liu YX, Zhao ZT, Gao Y, Jia CQ, Zhang JL, Yang ZQ, Wang SM, Jiang BF: Characterization of Orientia tsutsugamushi strains isolated in Shandong Province, China by immunofluorescence and restriction fragment length polymorphism (RFLP) analyses. Southeast Asian J Trop Med Public Health 2004, 35(2):353-357.

22. Tamura A, Takahashi K, Tsuruhara T, Urakami H, Miyamura S, Sekikawa H, Kenmotsu M, Shibata M, Abe S, Nezu H: Isolation of Rickettsia tsutsugamushi antigenically different from Kato, Karp, and Gilliam strains from patients. Microbiol Immunol 1984, 28(8):873-882.

23. Ikegaya S, Iwasaki H, Takada N, Yamamoto S, Ueda T: Tsutsugamushi disease caused by Shimokoshi-Type Orientia tsutsugamushi: the first report in Western Japan. Am J Trop Med Hyg 2013, 88(6):1217-1219.

\section{doi:10.1186/1756-3305-6-312}

Cite this article as: Zhang et al:: Molecular epidemiology of Orientia tsutsugamushi in chiggers and ticks from domestic rodents in Shandong, northern China. Parasites \& Vectors 2013 6:312

\section{Submit your next manuscript to BioMed Central and take full advantage of:}

- Convenient online submission

- Thorough peer review

- No space constraints or color figure charges

- Immediate publication on acceptance

- Inclusion in PubMed, CAS, Scopus and Google Scholar

- Research which is freely available for redistribution 